\title{
Supramolecular Chemistry to Improve Bioavailability of Phytochemicals
}

\section{Dinesh Kumar Yadav ${ }^{1 *}$, Pushpendra Koli ${ }^{2}$ and Kailash Pati Tripathi ${ }^{1}$}

${ }^{1}$ Ph. D. Scholar, Division of Agricultural Chemicals, ICAR-IARI, New Delhi, India

${ }^{2}$ Scientist, Indian Grassland and Fodder Research Institute, Jhansi, U.P, India

*Corresponding Author: Dinesh Kumar Yadav, Ph. D. Scholar, Division of Agricultural Chemicals, ICAR-IARI, New Delhi, India.

Received: August 26, 2019; Published: September 24, 2019

DOI: 10.31080/ASAG.2019.03.0664

\begin{abstract}
The usage of phytochemical supplements over past two decades has steadily increased worldwide. Popularity of these products has been especially notable among people in western countries, where almost $20 \%$ of consumers report regular usage of such supplements. Supramolecular interventions are being viewed with tremendous research inputs. Supramolecular chemistry refers to the area of chemistry beyond the molecules and focuses on the chemical systems made up of discrete number of assembled molecular subunits or components. The forces responsible for the spatial organization may vary and include mainly (intermolecular forces, electrostatic or hydrogen bonding). While traditional chemistry focuses on the covalent bond, supramolecular chemistry examines the weaker and reversible non-covalent interactions between molecules. In spite of the popularity of these phytochemical supplements, the actual efficacy has remained elusive for many of these supplements due to poor bioavailability. To improve the efficacy of these agents, researchers and manufacturers have been adopting novel drug formulation technologies to enhance phytochemical bioavailability. Cyclodextrins, cyclic oligosaccharides obtained from the degradation of starch, can be viable as an encapsulant for the plant bioactive compounds. Solubility and oral bioavailability of rutin (Flavanoid glycoside) was improved by complexation with HP- $\beta$-CD (2-hydroxypropyl- $\beta$-cyclodextrin). Phytosome ${ }^{\circledR}$ has been reported as an improved delivery system for oral bioavailability of polyphenolics through enhancement of rate and extent of solubility into aqueous intestinal fluids and capacity to cross bio membranes. Liposomes are extremely versatile supramolecules being developed and used as efficient carriers for drug delivery. Thus, improved phytochemical delivery through supramolecule chemistry may open up a new phase of operations in the "drug war" between humans and plants.
\end{abstract}

Keywords: Supramolecule; Phytochemicals; Bio-Availability; Phyto-Some and Liposome

\section{Introduction}

Supra (Latin) = above, beyond; Chemistry beyond the molecule, the science of non -covalent bond, intermolecular interactions".

\section{Definition}

"Supramolecular chemistry is the chemistry of the intermolecular bond, covering the structures and functions of the entities formed by the association of two or more chemical species" [4] (Figure 1).
- Host (receptor /Lock) is generally a large or macro-molecule having some sort of cavity which can hold a (generally) small molecule "guest", (substrate/key).

- Supramolecules form through non-covalent bonds - reversible

- Molecules form through covalent bonds - often irreversible 


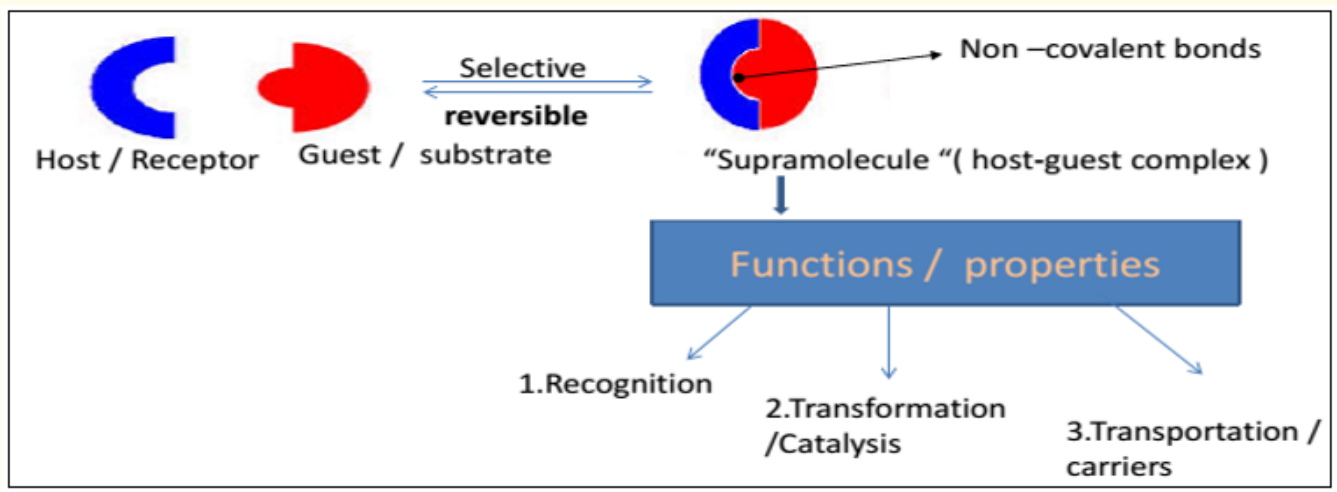

Figure 1: Host-guest complex/ supramolecular formation.

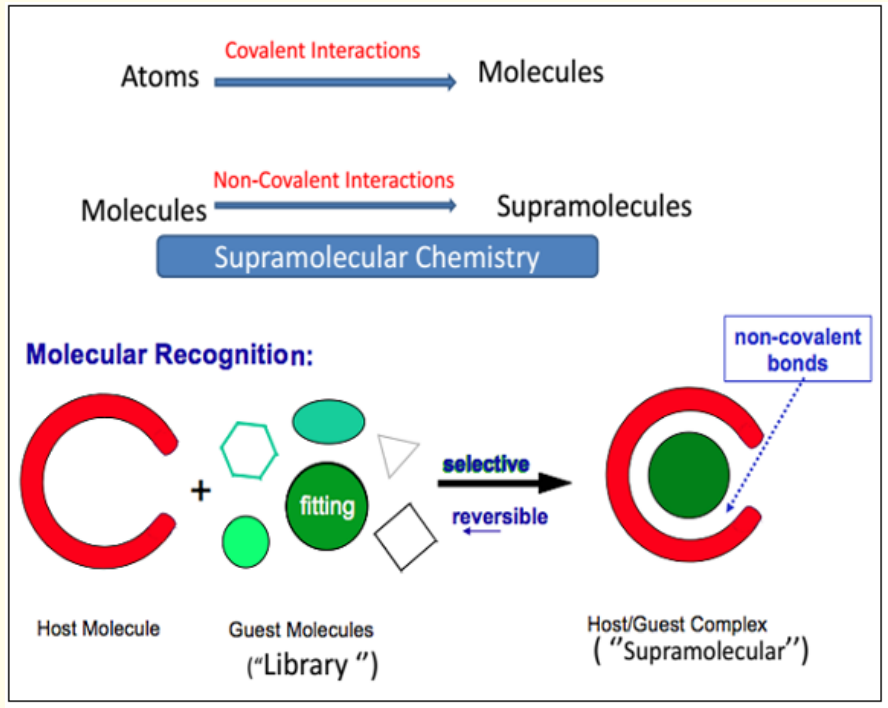

Figure 2: Host-guest complex interaction and product formation.

Approaches to enhance oral bioavailability of nutraceuticals $[1,7]$.

Protection of labile compounds

- Upon oral administration, nutraceuticals are subjected to complex digestion processes that involve changes in physiological or physiochemical environments. From the mouth to colon, the dynamic environmental conditions of GI tract may cause instability to the chemical structures of active ingredients.

- The factors, such as $\mathrm{pH}$ variations, ionic strength, enzyme degradations, mechanistic motilities etc., all potentially contribute to the degradation of nutraceuticals. Therefore, delivery systems which are designed with protective mechanism could enhance the gastric stability of labile bioactive nutrients and, thus, the oral dosing efficiency.

\section{Extension of gastric retention time}

- Gastrointestinal digestion is a dynamic moving process, in which the nutrients are continuously pushed down to subsequent digestion and absorption sites.

- Insufficient gastric retention time results in the incomplete absorption of nutrients, excessive compound excretion, and decrease in the dose responsive efficiency of therapeutic purposes. 
- Formulating delivery systems with higher viscosity or ability to slow down the gastric movement of bioactive compounds prolongs the residence time in the GI tract and allows higher percentage of bioactives to be absorbed before gastric emptying.

Increase aqueous solubility

- In order to be effectively taken up by the intestinal enterocytes, the bioactive compounds need to be either solubilized or suspended or dispersed in the aqueous environment.

- Unlike hydrophilic nutrients, compounds which are lipophilic tend to have poor solubility and frequently precipitate as clusters when added to the aqueous environment

- Large compound clusters inhibit intestinal absorptions, which withhold a critical particle size requirement, and are rapidly eliminated through the excretion mechanisms. Since poor aqueous solubility is a major factor which limits the absorption of lipophilic compounds, delivery systems which are capable of enhancing solubility or dispersion of such ingredients can effectively augment their concentrations getting into the targeted action site within the biological systems.

Controlled/delayed release

- The maintenance of constant dosage level within the systemic circulation is one of the important factors to sustain meaningful physiological efficacy between the therapeutic intervals. The well-designed controlled release mechanisms are capable of sustaining constant release profiles which can best comply with the digestion and absorption processes.

- In order for the contained compound to be released from the delivery vehicle, the delivery vehicles need to be digested or disintegrated by enzymatic activity. The release rate and time of bioactive compounds can be controlled through selecting materials with different digestive sustainability and incorporating layers of digestive protection material on vehicle surfaces.

\section{Modulation of metabolic activities}

- While the absorption sets the first barrier of the bioavailability, the first-pass metabolic activities are the second hurdle to reduce the systemic dosage level of nutraceuticals.

- The inclusion of physical or chemical inhibitor of metabolic enzymes in the delivery systems may significantly enhance the concentration level of bioactives in the systemic circulation. However, the utilization of such enzyme inhibitor may need more careful consideration to avoid toxicity raised due to impaired detoxification activity

\section{Causes of poor bioavailability}

- Rapid metabolism

- Extensive presystemic metabolism

- Large molecular size

- Poor water solubility

- Poor miscibility with oils and other lipids, which limited their ability to pass across the lipid-rich membranes

Technologies for improving phytochemical bioavailability

1. Liposome; 2. Phytosome; 3. Encapsulating Agent.

\section{Liposome}

- Liposome is a tiny bubble (vesicle), made out of the same material as a cell membrane.

- Liposomes are microscopic artificial vesicles composed of phospholipid membranes surrounding an aqueous core.

- Membranes are usually made of phospholipids, which are molecules that have a hydrophilic head group and a hydrophobic tail group.

- Phosphatidylcholine molecules surround the water-soluble substance.

- $\quad$ No chemical bond is formed [3].

\section{Phytosome}

- Phyto constituents (mainly polyphenolics) can be converted into a lipid-compatible molecular complex known as PHYTOSOME®. A PHYTOSOME $®$ is generally more bioavailable than a simple herbal extract due to its enhanced capacity to cross the lipid-rich biomembranes [2].

- $\quad$ PHYTOSOME $^{\circledR}$ is not a mechanical mixture of two constituents, but a specific complex between a hydrophilic guest and a lipophilic host. The phospholipid mainly employed to make phytosomes, is phosphatidylcholine, derived from soybean (Glycine max).

- Phosphatidylcholine, an essential part of the cell membrane used in phytosome technology, acts as a carrier. Phytosome process the phosphatidylcholine and the individual plant components actually from a 1:1 or a 2:1 complex depending on the substance 
Difference between phytosome and liposome

- The fundamental difference between liposomes and phytosomes is that in liposomes the active principle is dissolved in the medium contained in the cavity or in the layers of the membrane, whereas in the phytosome it is an integral part of the membrane, being the molecules anchored through chemical bonds to the polar head of the phospholipid.

- A liposome is formed by mixing a water-soluble substance with phosphatidylcholine. No chemical bond is formed; the phosphatidylcholine molecules surround the water-soluble substance. There may be hundreds or even thousands of phosphatidylcholine molecules surrounding the water-soluble compound. In contrast, with the Phytosome process the phosphatidylcholine and the individual plant components actually from a 1:1 or a 2:1 complex depending on the substance (Figure 3).

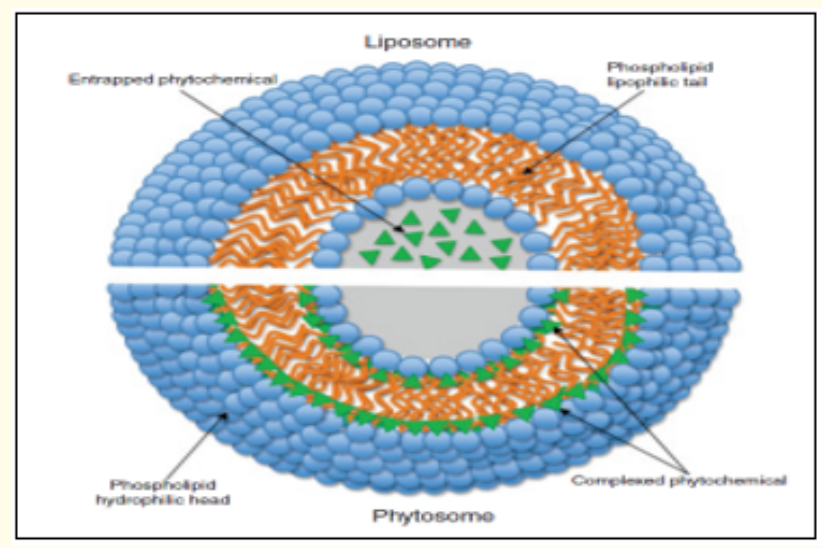

Figure 3: Phytosome and liposome.

\section{Encapsulating Agent (Cyclodextrins)}

- Cyclodextrins are structurally related natural products, they belong to the family of cyclic oligosaccharides with a hydrophilic outer surface and a lipophilic central cavity Cyclodextrins are cyclic oligosaccharides arising from the degradation of starch, which can be a viable option as encapsulation technique.

- CDs are cyclic oligosaccharides with 6, 7 or 8 glucose unit linked by (1-4) glycosidic bond.CD molecules are cone shape, with a hydrophobic zone inside and a hydrophilic external surface $[5,6]$.
- CDS also known as Schardinge dextrin's and this CDS contain (1,4)-linked D-glucopyranose units. Cyclodextrins are inexpensive, friendly to humans, and also capable of improving the biological, chemical and physical properties of bioactive molecules.

\section{Conclusion}

Supramolecular (Cyclodextrins, Phytosome, Liposome etc.) can serves a promising pathway for the development of pharmaceutical products to the user. These molecules are inexpensive, friendly to humans, and also capable of improving the biological, chemical and physical properties of bioactive molecules. In addition, these are extremely versatile leading to a large number of applications in pharmaceutical, cosmetics and food industrial fields.

\section{Bibliography}

1. Bailey R L., et al. "Dietary supplement use in the United States, 2003-2006". The Journal of Nutrition 141.2 (2010): 261-266.

2. Kidd P M. "Bioavailability and activity of phytosome complexes from botanical polyphenols: the silymarin, curcumin, green tea, and grape seed extracts". Alternative Medicine Review 14.3 (2009): 226-246.

3. Laouini A., et al. "Preparation, characterization and applications of liposomes: State of the art". Journal of Colloid Science and Biotechnology 1 (2012): 147-168.

4. Lehn J M. "Supramolecular chemistry". Science 260 (1993): 1762-1763.

5. Miyake K., et al. "Improvement of solubility and oral bioavailability of rutin by complexation with 2-hydroxypropyl- $\beta$ cyclodextrin". Pharmaceutical Development and Technology 5 (2000): 399-407.

6. Pinho E., et al. "Cyclodextrins as encapsulation agents for plant bioactive compounds". Carbohydrate Polymers 101 (2014): 121-135.

7. Weber P. "Bioactive compounds: Safety and efficacy". Nutrition 25 (2009): 1206-1211.

Volume 3 Issue 10 October 2019 (C) All rights are reserved by Dinesh Kumar Yadav., et al. 